\title{
ON THE EXPRESSIBILITY OF A UNIFORM FUNCTION OF SEVERAL COMPLEX VARIABLES AS THE QUOTIENT OF TWO FUNCTIONS OF ENTIRE CHARACTER*
}

BY

\section{T. H. GRONWALL}

\section{INTRODUCTION}

It is a classical fact in the theory of functions of one complex variable that any meromorphic function may be expressed as the quotient of two entire functions without common zeros. When $f(x)$ is a uniform function with essential singularities at finite distance, this theorem may be extended, as was shown by Weierstrass $\dagger$ for a finite number of essential singularities, and by Mittag-Leffler in the general case: $f(x)$ is expressible as the quotient of two functions of entire character (that is, uniform and without poles, but generally both having the same essential singularities as $f(x)$ ) without common zeros.

Before taking up the corresponding question for several variables, it is convenient to recall the following definitions:

The complex variables $x_{1}, x_{2}, \cdots, x_{i 2}$ are interior to the region $\left(S_{1}, S_{2}\right.$, $\cdots, S_{n}$ ) when $x_{1}$ is interior to the region $S_{1}$ in the $x_{1}$-plane, $\cdots, x_{n}$ interior to the region $S_{n}$ in the $x_{n}$-plane; the regions $S_{1}, \cdots, S_{n}$ may be simply or multiply connected.

A uniform function $f\left(x_{1}, x_{2}, \cdots, x_{n}\right)$ of the complex variables $x_{1}, x_{2}, \cdots, x_{n}$ is meromorphic in $\left(S_{1}, S_{2}, \ldots, S_{n}\right)$ when, in the vicinity of every point $a_{1}, a_{2}, \cdots, a_{n}$ interior to $\left(S_{1}, S_{2}, \cdots, S_{n}\right)$, we have

$$
f\left(x_{1}, x_{2}, \cdots, x_{n}\right)=\frac{P_{1}\left(x_{1}-a_{1}, x_{2}-a_{2}, \cdots, x_{n}-a_{n}\right)}{P_{0}\left(x_{1}-a_{1}, x_{2}-a_{2}, \cdots, x_{n}-a_{n}\right)},
$$

where $P_{0}$ and $P_{1}$ are power series in $x_{1}-a_{1}, x_{2}-a_{2}, \cdots, x_{n}-a_{n}$. A uniform function $G\left(x_{1}, x_{2}, \cdots, x_{n}\right)$ is of entire character in $\left(S_{1}, S_{2}, \cdots, S_{n}\right)$

* Presented to the Society, October 25, 1913.

$\dagger \mathrm{K}$. Weierstrass, Zur Theorie der eindeutigen analytischen Fuintionen, iMathematische Werke, vol. 2 (Berlin, 1895), pp. 77-124. G. Mittá-Leffler, Nur la représentation analytique des fonctions monogènes uniformes d'une variable indépendunie, $\mathrm{A} \mathrm{ct} \mathrm{a} \mathrm{M}$ a t $\mathrm{h}$ e $\mathrm{mat} \mathrm{ica}$, vol. 4 (1884), pp. 1-79. 
when holomorphic at every point $a_{1}, a_{2}, \cdots, a_{n}$ interior to $\left(S_{1}, S_{2}, \cdots, S_{n}\right)$. Two functions of entire character $G_{0}\left(x_{1}, x_{2}, \cdots, x_{n}\right)$ and $G_{1}\left(x_{1}, x_{2}, \cdots, x_{n}\right)$ have a common divisor when there exists a point $a_{1}, a_{2}, \ldots, a_{n}$ interior to $\left(S_{1}, S_{2}, \cdots, S_{n}\right)$ such that in its vicinity

$$
\begin{aligned}
& G_{0}\left(x_{1}, x_{2}, \cdots, x_{n}\right)=P\left(x_{1}-a_{1}, x_{2}-a_{2}, \cdots, x_{n}-a_{n}\right) \\
& \cdot P_{0}\left(x_{1}-a_{1}, x_{2}-a_{2}, \cdots, x_{n}-a_{n}\right), \\
& G_{1}\left(x_{1}, x_{2}, \cdots, x_{n}\right)=P\left(x_{1}-a_{1}, x_{2}-a_{2}, \cdots, x_{n}-a_{n}\right) \\
& \cdot P_{1}\left(x_{1}-a_{1}, x_{2}-a_{2}, \cdots, x_{n}-a_{n}\right),
\end{aligned}
$$

with $P(0,0, \cdots, 0)=0$. Two functions of entire character are relatively prime when they have no common divisor.

Poincaré has shown,* by the theory of harmonic functions of four real variables, that when $n=2$ and $S_{1}$ and $S_{2}$ contain all points at finite distance in the $x_{1-}$ and $x_{2}$-planes respectively, every meromorphic function is expressible as the quotient of two entire functions without common divisor. In a later paper, $\dagger$ he has modified this method and extended it to $n$ variables.

The Cauchy integral was used by Cousinł to prove Poincarés result and extend it to more general regions. His most general results are the following, of which A may be regarded as the extension to several variables of MittagLeffler's theorem, while B generalizes Weierstrass's theorem on the existence of uniform functions with given zeros:

A. When for every point $a_{1}, a_{2}, \cdots, a_{n}$ interior to $\left(S_{1}, S_{2}, \cdots, S_{n}\right)$ there are given

(1) a region $\Gamma_{a_{1}, a_{2}}, \cdots, a_{n}$ consisting of $n$ circles $\left|x_{\nu}-a_{\nu}\right|<r_{\nu}(\nu=1,2$, $\cdots, n)$, each of these circles being interior to the corresponding region $S_{v}$;

(2) a function $f_{a_{1}, a_{2}}, \cdots, a_{n}\left(x_{1}, x_{2}, \cdots, x_{n}\right)$ uniform in $\Gamma_{a_{1}, a_{2}}, \cdots, a_{n}$ and such that when two regions $\Gamma_{a_{1}}, a_{2}, \cdots, a_{n}$ and $\Gamma_{a_{1}^{\prime}, a_{2}^{\prime}, \ldots, a_{n}^{\prime}}$ have a region in common, the difference

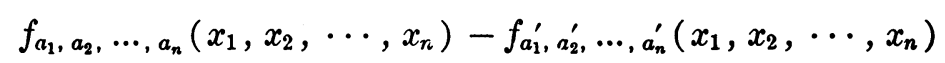

is holomorphic in the common region;

Then there exists a function $F\left(x_{1}, x_{2}, \cdots, x_{n}\right)$ uniform in $\left(S_{1}, S_{2}, \cdots, S_{n}\right)$ and such that for every interior point $a_{1}, a_{2}, \cdots, a_{n}$ the difference

$$
F\left(x_{1}, x_{2}, \cdots, x_{n}\right)-f_{a_{1}, a_{2}}, \cdots, a_{n}\left(x_{1}, x_{2}, \cdots, x_{n}\right)
$$

is holomorphic in $\Gamma_{a_{1}}, a_{2}, \cdots, a_{n}$.

${ }^{*} \mathrm{H}$. Poincaré, Sur les fonctions de deux variables, A c t a M a the matica, vol. 2 (1883), pp. 97-113.

† H. Poincaré, Sur les propriétés du potentiel et sur lesfonctions Abéliennes, A c t a $\mathrm{M}$ a $\mathrm{t}$ h em a t i c a, vol. 22 (1899), pp. 89-178.

$\ddagger \mathrm{P}$. Cousin, Sur les fonctions de $n$ variables complexes, A $\mathrm{ct}$ a $\mathrm{M}$ a th $\mathrm{e} \mathrm{m}$ a ti $\mathrm{c}$ a, vol. 19 (1895), pp. 1-62. 
B. When for every point $a_{1}, a_{2}, \cdots, a_{n}$ interior to $\left(S_{1}, S_{2}, \cdots, S_{n}\right)$ there are given

(1) a region $\Gamma_{a_{1}, a_{3}}, \cdots, a_{n}$ as in $\mathbf{A}$;

(2) a function $u_{a_{1}, a_{2}, \cdots, a_{n}}\left(x_{1}, x_{2}, \cdots, x_{n}\right)$ of entire character in $\Gamma_{a_{1}, a_{2}}, \ldots, a_{n}$ and such that when two regions $\Gamma_{a_{1}, a_{2}}, \ldots, a_{n}$ and $\Gamma_{a_{1}^{\prime}, a_{2}^{\prime}, \cdots, a^{\prime}}$ have a region in common, the quotient

$$
u_{a_{1}, a_{2}, \cdots, a_{n}}\left(x_{1}, x_{2}, \cdots, x_{n}\right) / u_{a_{1}^{\prime}, a_{3}^{\prime}, \cdots, a_{n}^{\prime}}\left(x_{1}, x_{2}, \cdots, x_{n}\right)
$$

is holomorphic and different from zero in the common region;

Then there exists a function $G\left(x_{1}, x_{2}, \cdots, x_{n}\right)$ of entire character in $\left(S_{1}, S_{2}, \cdots, S_{n}\right)$ such that for every interior point $a_{1}, a_{2}, \cdots, a_{n}$ the quotient $G\left(x_{1}, x_{2}, \cdots, x_{n}\right) / u_{a_{1}}, a_{2}, \cdots, a_{n}\left(x_{1}, x_{2}, \cdots, x_{n}\right)$ is holomorphic and different from zero in $\Gamma_{a_{1}, a_{2}}, \cdots, a_{n}$.

C. When a function $f\left(x_{1}, x_{2}, \cdots, x_{n}\right)$ is meromorphic in $\left(S_{1}, S_{2}, \cdots, S_{n}\right)$, it may be expressed as the quotient of two relatively prime functions of entire character* in $\left(S_{1}, S_{2}, \cdots, S_{n}\right)$ :

$$
f\left(x_{1}, x_{2}, \cdots, x_{n}\right)=\frac{G_{1}\left(x_{1}, x_{2}, \cdots, x_{n}\right)}{G_{0}\left(x_{1}, x_{2}, \cdots, x_{n}\right)} .
$$

Cousin establishes Theorem $\mathbf{A}$ in its various stages in an entirely rigorous manner, but his proofs of Theorem B (and hence of Theorem C, which is a quite elementary consequence of B-see Cousin, l. c., $\$ \S 15,19$, and 25) contain a gap (at stages $\alpha$ and $\beta$ ) which considerably restricts the regions $\left(S_{1}, S_{2}, \cdots, S_{n}\right)$ in which they are applicable.

In $\S 2$, the nature of this gap is explained, and Cousin's proofs of $\mathbf{B}$ are shown to be valid when all, or all but one, of the $n$ regions $S_{1}, S_{2}, \cdots, S_{n}$ are simply connected. On the other hand, it is established by an example that Cousin's construction of $G\left(x_{1}, x_{2}, \cdots, x_{n}\right)$ does not always yield a uniform function when two of the regions $S_{1}, S_{2}, \ldots, S_{n}$ are multiply connected.

The question now arises as to the validity of Theorems $\mathbf{B}$ and $\mathbf{C}$ in the cases where Cousin's proofs do not apply. In $\S 3$ it is shown by an example that Theorem $\mathbf{C}$ is false (and consequently Theorem B, since $\mathbf{C}$ would follow from B) when two of the regions $S_{1}, S_{2}, \cdots, S_{n}$ are multiply connected, that is, in the very cases where Cousin's proofs fail.

${ }^{*}$ In his proofs, Cousin proceeds by four stages: first the theorems are derived for any region $\left(s_{1}, s_{2}, \cdots, s_{n}\right)$ interior to $\left(S_{1}, S_{2}, \cdots, S_{n}\right)$, and this separately for $n=2$ (stage $\alpha$ ) and $n$ general (stage $\beta$ ). Second, a limiting process is used to extend the region of validity of the theorems from $\left(s_{1}, s_{2}, \cdots, s_{n}\right)$ to $\left(S_{1}, S_{2}, \cdots, S_{n}\right)$, and this separately when all $S_{\nu}$ are circles (stage $\gamma$ ) and when $S_{\nu}$ are quite general (stage $\delta$ ). For convenient reference, the numbers of Cousin's theorems corresponding to Theorems A, B, and C of the text at the various stages are given below:

\begin{tabular}{lllll} 
& $\alpha$ & \multicolumn{1}{c}{$\beta$} & \multicolumn{1}{c}{$\gamma$} \\
A & I & IV & VII, p. 33 & XI \\
B & III & VI & IX & XII \\
C & - & VII,, p. 32 & X & XIV
\end{tabular}


Thus the results of the present paper may be summarized in the statement that

Theorems $\mathrm{B}$ and $\mathrm{C}$ are valid when, and only when, $n-1$ of the $n$ regions $S_{1}, S_{2}, \cdots, S_{n}$ are simply connected; the remaining region may be simply or multiply connected.

The author wishes to acknowledge his indebtedness to Professor Osgood, to whom he communicated the example of $\S 3$ in June, 1913, for material assistance in locating the gap in Cousin's proofs.

\section{The domain of validity of Cousin's proofs of Theorem B}

To abridge the notation, we shall write $x$ for the system of $n-1$ variables $x_{1}, x_{2}, \cdots, x_{n-1}$ and $S$ for $\left(S_{1}, S_{2}, \cdots, S_{n-1}\right) ; x_{n}$ will be denoted by $y$ and $S_{n}$ by $S^{\prime}$. A simply connected part $\Sigma$ of $S$ we define as a system of regions $\left(\Sigma_{1}, \Sigma_{2}, \cdots, \Sigma_{n-1}\right)$ where, for $\nu=1,2, \cdots, n-1$, every interior or boundary point of the simply connected region $\Sigma_{\nu}$ is interior to or on the boundary of $S_{\nu}$. The boundaries of $S_{1}, S_{2}, \cdots, S_{n-1}, \Sigma_{1}, \Sigma_{2}, \cdots, \Sigma_{n-1}$, and $S^{\prime}$ are assumed to be regular, that is, each is to consist of a finite number of pieces of analytic curves without singular points.

We now assume $S^{\prime}$ to be subdivided, by a finite number of pieces of regular curves, into a finite number of simply connected regions $R_{1}, R_{2}, \cdots, R_{n}, \cdots$. When $R_{n}$ and $R_{p}$ are adjacent regions, we denote by $l_{n p}$ their common boundary, or, should this consist of several pieces, any one of these. If any $l_{n p}$ is a closed curve, we cut it at three points, thus obtaining three pieces such that no two of them taken together form a closed curve. The direction of $l_{n p}$ is that which leaves the interior of the region $R_{n}$ to the left, so that $l_{n p}$ and $l_{p n}$ are the same curve described in opposite directions. Finally, let $T_{n p}$ consist of all points in the $y$-plane interior to at least one circle with center on $l_{n p}$ and sufficiently small radius $r$, this $r$ being constant not only for different points on $l_{n p}$, but also for all the various curves $l_{n p}$.

The proof of Theorem B now depends on the following lemma:

Let a function $u_{p}(x, y)$ be given for every region $R_{p}$, uniform and holomorphic in $\left(S, R_{p}\right)$, boundaries included, and such that for any two adjacent regions $R_{n}$ and $R_{p}$, the quotient

$$
\frac{u_{p}(x, y)}{u_{n}(x, y)}=g_{n p}(x, y)
$$

is holcmorphic and different from zero in $\left(S, T_{n p}\right)$. Then there exists a function $G(x, y)$ holomorphic in $\left(S, S^{\prime}\right)$, uniform in $\left(\Sigma, S^{\prime}\right)$, where $\Sigma$ is any simply connected part of $S$, and such that in $\left(S, R_{p}\right)$ (boundaries included, except those $y$ unich are end points of an $l_{n p}$ and lie cn the boundary of $S^{\prime}$ ) the 
quotient

$$
\frac{G(x, y)}{u_{p}(x, y)}
$$

is holomorphic and different from zero.

When $S$ is simply connected, we may evidently let $\Sigma$ coincide with $S$. In his formulation of the lemma (l. c., $\S 7$; proof in $\S 6$ ) Cousin makes no distinction between $\Sigma$ and $S$, so that, when $S$ is multiply connected (that is, one at least of $S_{1}, S_{2}, \cdots, S_{n-1}$ is multiply connected) he tacitly assumes the function $G(x, y)$ to be uniform in $\left(S, S^{\prime}\right)$, while the uniformity is proved only in $\left(\Sigma, S^{\prime}\right)$.

This constitutes the gap in Cousin's proofs referred to in the introduction. It might also be objected to his proof of the lemma (l. c., § 6 ) that he operates throughout with the multiform functions $\log u_{p}(x, y)$ and their differences $\log u_{p}(x, y)-\log u_{n}(x, y)$, and that it is not quite clear what branches of these functions are meant at the various points of $\left(S, S^{\prime}\right)$; but this objection is met by a modification of Cousin's argument due to Osgood.*

Since $u_{p}(x, y)$ and $u_{n}(x, y)$ are uniform in $\left(S, T_{n p}\right)$ by hypothesis, and their quotient $g_{n p}(x, y)$ is holomorphic and different from zero in the same region, it follows that writing

$$
G_{n p}(x, y)=\log g_{n p}(x, y),
$$

where that branch of $\log g_{n p}(x, y)$ is taken which assumes its principal value at some point $x_{0}, y_{0}$ interior to $\left(\Sigma, T_{n p}\right)$, the function $G_{n p}(x, y)$ is holomorphic in $\left(S, T_{n p}\right)$ and uniform in $\left(\Sigma, T_{n p}\right)$. Next let

$$
I_{n p}(x, y)=\frac{1}{2 \pi i} \int_{l_{n p}}^{\cdot} \frac{G_{n p}(x, z) d z}{z-y},
$$

the integral being taken in the positive direction of $l_{n p}$. This function is holomorphic for all $y$ at finite or infinite distance, except those on the curve $l_{n p}$, and for any $x$ in $S$, and uniform for the same $y$ and any $x$ in $\Sigma$. Moreover, as shown in Cousin $\$ \S 2-3$,

$$
\begin{aligned}
& I_{n p}(x, y)=H(x, y)+G_{n p}(x, y) \lambda_{n p}(y), \\
& \lambda_{n p}(y)=\frac{1}{2 \pi i} \log \frac{y-b}{y-a}, \quad \lambda_{n p}(\infty)=0,
\end{aligned}
$$

where $a$ and $b$ are the end points of $l_{n p}, \log [(y-b) /(y-a)]$ is that branch of the logarithm which vanishes for $y=\infty$, so that $\lambda_{n p}(y)$ is uniform and holomorphic in the whole $y$-plane except on the curve $l_{n p}$, and finally $H(x, y)$

* Letter to the author, July 7, 1913. This modified proof is reproduced here with the permission of Professor Osgood. 
is holomorphic in $\left(S, T_{n p}\right)$ and uniform in $\left(\Sigma, T_{n p}\right)$. Now write

$$
\Phi(x, y)=\sum I_{n p}(x, y),
$$

where the summation is extended over all the curves $l_{n p}$ which are common to the boundaries of two regions $R$ (each curve taken once, and not in the two subscript combinations $l_{n p}$ and $\left.l_{p n}\right)$, and define

$$
\phi_{n}(x, y)=\Phi(x, y) \text { in }\left(S, R_{n}\right) \text {. }
$$

Then $\phi_{n}(x, y)$ is holomorphic in $\left(S, R_{n}\right)$ and uniform in $\left(\Sigma, R_{n}\right)$, boundaries included except the end points of the various $l_{n p}$ belonging to the boundary of $R_{n}$. Denoting by $\phi_{n}(x, y)_{p}$ the analytic continuation of $\phi_{n}(x, y)$ when $x$ describes any path in $S$ and $y$ a path in $T_{n p}$ starting at a point inside $R_{n}$ and ending at a point inside $R_{p}$, but not passing through an end point of $l_{n p}$, we have (Cousin, l. c., $\$ \$ 2-3$ )

$$
\phi_{n}(x, y)_{p}=\phi_{p}(x, y)+G_{n p}(x, y) \text {. }
$$

A point $y=b$ interior to $S^{\prime}$ is called a vertex when it is an end point of any $l_{n p}$. Now make

$$
\bar{G}_{n}(x, y)=u_{n l}(x, y) e^{\phi_{n}(x, y)} \text { in }\left(S, R_{n}\right) \text {; }
$$

then it follows from (1) that $\bar{G}_{p}(x, y)$ is the analytic continuation of $\bar{G}_{n}(x, y)$ across $l_{n p}$ (the path in the $y$-plane leading from $R_{n}$ into $R_{p}$ not crossing $l_{n p}$ at a vertex), and consequently the continuation of $\bar{G}_{n}(x, y)$ along a closed path in the $y$-plane not passing through any vertex brings us back to $G_{n}(x, y)$. We may therefore define a single function $\bar{G}(x, y)$ by the consistent conditions $\bar{G}(x, y)=\bar{G}_{n}(x, y)$ in $\left(S, R_{n}\right)$, and this $\bar{G}(x, y)$ is visibly uniform in $\left(\Sigma, S^{\prime}\right)$. Moreover, the quotient $\bar{G}(x, y) / u_{p}(x, y)$ is holomorphic and different from zero in $\left(S, R_{p}\right)$, boundaries included, except when $y$ coincides with an end point of an $l_{n p}$ while $x$ takes any value inside or on the boundary of $S$.

We shall now modify $\bar{G}(x, y)$ so as to remove the last restriction for those end points of an $l_{n p}$ which are vertices. Let $b$ be a vertex, and suppose that, for instance, $R_{1}, R_{2}, \cdots, R_{m}$ are those regions $R$ which are adjacent to this vertex. Let $1 \leqq \nu \leqq m$ and denote by $R_{\nu}^{\prime}$ that part of $R_{\nu}$ which lies within or on the circle $|y-b|=r^{\prime}$, where $r^{\prime}$ is less than the radius $r$ of the circles used in defining all $T_{n p}$. Then we have in $\left(S, R_{\nu}^{\prime}\right)$

$$
\begin{aligned}
\phi_{\nu}(x, y)=\Phi(x, y) & =A(x, y)+G_{12}(x, y) \lambda_{12}(y)+G_{23}(x, y) \lambda_{23}(y) \\
+ & \cdots+G_{m-1, m}(x, y) \lambda_{m-1, m}(y)+G_{m 1}(x, y) \lambda_{m 1}(y),
\end{aligned}
$$

$A(x, y)$ being holomorphic in $\left(S,|y-b| \leqq r^{\prime}\right)$ and uniform in $(\Sigma,|y-b|$ $\left.\leqq r^{\prime}\right)$. Make

$$
L_{\nu}(y-b)=\frac{1}{2 \pi i} \log (y-b)
$$


where any branch of the logarithm is chosen and rendered uniform by a cut issuing from $y=b$, but having no other point in common with $R_{\nu}^{\prime}$ or its boundary. None of the $l_{n p}$ abutting at $b$ being closed, we may continue $\lambda_{n p}(y)$ analytically from $y=\infty$ to a point inside $R_{\nu}^{\prime}$ along a curve intersecting none of these $l_{n p}$, and in the relation

$$
\lambda_{n p}(y)-L_{\nu}(y-b)=-\frac{1}{2 \pi i} \log (y-a),
$$

where now $\log (y-a)$ is a definite branch of the logarithm, for $y$ in $R_{v}^{\prime}$, the right-hand member is holomorphic in the entire region $|y-b| \leqq r^{\prime}$. Hence we have, for $y$ interior to $R_{v}^{\prime}$,

$$
\begin{aligned}
\phi_{\nu}(x, y)=B_{\nu}(x, y)+\left[G_{12}(x, y)\right. & +G_{23}(x, y)+\cdots \\
& \left.+G_{m-1, m}(x, y)+G_{m 1}(x, y)\right] L_{\nu}(y-b),
\end{aligned}
$$

where $B_{v}(x, y)$ is holomorphic in $\left(S,|y-b| \leqq r^{\prime}\right)$ and uniform in ( $\Sigma$, $\left.|y-b| \leqq r^{\prime}\right)$. On the other hand, the sum in brackets equals

$$
\log \frac{u_{2}(x, y)}{u_{1}(x, y)}+\log \frac{u_{3}(x, y)}{u_{2}(x, y)}+\cdots+\log \frac{u_{m}(x, y)}{u_{m-1}(x, y)}+\log \frac{u_{1}(x, y)}{u_{m}(x, y)},
$$

where each log refers to a definite branch of the function-the branch chosen at the beginning, and this sum therefore equals a definite value of $\log 1$, which we denote by $2 \pi i K_{b}$, the integer $K_{b}$ being evidently independent of $\nu$. Consequently, for $y$ interior to $R_{\nu}^{\prime}$,

$$
(y-b)^{-K_{b}} \bar{G}(x, y)=u_{\nu}(x, y) e^{\phi_{\nu}(x, y)-2 \pi i K_{b} L_{\nu}(y-b)},
$$

or

$$
(y-b)^{-K_{b}} \bar{G}(x, y)=u_{\nu}(x, y) e^{B_{\nu}(x, y)}
$$

but the expression to the right being holomorphic in $\left(S,|y-b| \leqq r^{\prime}\right)$ and uniform in $\left(\Sigma,|y-b| \leqq r^{\prime}\right)$, it follows by analytic continuation that the same is true of the left-hand member, and that the quotient of the latter by $u_{\nu}(x, y)$, which equals $e^{B_{\nu}(x, y)}$ in $\left(S, R_{\nu}^{\prime}\right)$, is holomorphic and different from zero in that region.

Finally determine the integer $K_{b}$ for each vertex $b$ and write

$$
G(x, y)=\bar{G}(x, y) \prod_{b}(y-b)^{-K_{b}},
$$

the product extending over all vertices. It then follows immediately from the preceding argument that $G(x, y)$ has all the properties mentioned in the lemma.

As already stated, Cousin tacitly assumes that from the proven uniformity of $G(x, y)$ in $\left(\Sigma, S^{\prime}\right)$ it follows that $G(x, y)$ is also uniform in $\left(S,^{\prime}\right) S$ when $S$ is multiply connected. 
I shall now show by an example that this conclusion is not legitimate; it is evidently sufficient to assume $n=2$, so that now $x$ stands for a single variable, and $S$ for a region in the $x$-plane. This example, as well as the one in $\S 3$, is based on the simplest properties of Theta functions of two variables. It is well known that, given the constants $\tau_{11}, \tau_{12}, \tau_{22}$ such that the real part of $2 \pi i\left(\tau_{11} n_{1}^{2}+2 \tau_{12} n_{1} n_{2}+\tau_{22} n_{2}^{2}\right)$ is a definite negative quadratic form in $n_{1}$ and $n_{2}$, the two expressions*

$$
\phi_{\nu}\left(v_{1}, v_{2}\right)=\sum_{n_{1}, n_{2}=-\infty}^{+\infty} \operatorname{Exp}\left[\left(n_{1}-\frac{\nu}{2}\right)^{2} \tau_{11}+2\left(n_{1}-\frac{\nu}{2}\right) n_{2} \tau_{12}\right.
$$

$$
\left.+n_{2}^{2} \tau_{22}-2\left(n_{1}-\frac{\nu}{2}\right) v_{1}-2 n_{2} v_{2}\right]
$$

where $\nu=0$ or 1 , define entire functions of $v_{1}$ and $v_{2}$ with the properties

$$
\begin{aligned}
\phi_{\nu}\left(v_{1}+1, v_{2}\right) & =\phi_{\nu}\left(v_{1}, v_{2}\right), \\
\phi_{\nu}\left(v_{1}, v_{2}+\frac{1}{2}\right) & =\phi_{\nu}\left(v_{1}, v_{2}\right), \\
\phi_{\nu}\left(v_{1}+\tau_{11}, v_{2}+\tau_{12}\right) & =\operatorname{Exp}\left(-2 v_{1}-\tau_{11}\right) \cdot \phi_{\nu}\left(v_{1}, v_{2}\right), \\
\phi_{\nu}\left(v_{1}+\tau_{12}, v_{2}+\tau_{22}\right) & =\operatorname{Exp}\left(-2 v_{2}-\tau_{22}\right) \cdot \phi_{\nu}\left(v_{1}, v_{2}\right)
\end{aligned}
$$

Assume $\tau_{12} \neq 0$, introduce new variables $w_{1}$ and $w_{2}$ by the relations

$$
\tau_{12} w_{1}=-2 \tau_{22} v_{1}+2 \tau_{12} v_{2}, \quad \tau_{12} w_{2}=v_{1},
$$

and write $\phi_{\nu}\left(v_{1}, v_{2}\right)=\psi_{\nu}\left(w_{1}, w_{2}\right)$; then $\psi_{\nu}\left(w_{1}, w_{2}\right)$ are entire functions of $w_{1}$ and $w_{2}$ with the properties

$$
\begin{gathered}
\psi_{\nu}\left(w_{1}+1, w_{2}\right)=\psi_{\nu}\left(w_{1}, w_{2}\right) \\
\psi_{\nu}\left(w_{1}, w_{2}+1\right)=\operatorname{Exp}\left(-w_{1}-2 \tau_{22} w_{2}-\tau_{22}\right) \cdot \psi_{\nu}\left(w_{1}, w_{2}\right),
\end{gathered}
$$$$
\quad \psi_{\nu}\left(w_{1}-\frac{2 \tau_{22}}{\tau_{12}}, w_{2}+\frac{1}{\tau_{12}}\right)=\psi_{\nu}\left(w_{1}, w_{2}\right), \quad(\nu=0,1) \text {. }
$$

$$
\psi_{\nu}\left(w_{1}+\frac{2 \tau_{12}^{2}-2 \tau_{11} \tau_{22}}{\tau_{12}}, w_{2}+\frac{\tau_{11}}{\tau_{12}}\right)=\operatorname{Exp}\left(-2 \tau_{12} w_{2}-\tau_{11}\right) \cdot \psi_{\nu}\left(w_{1}, w_{2}\right)
$$

Finally write $\psi\left(w_{1}, w_{2}\right)=\operatorname{Fxp}\left(\tau_{22} w_{2}^{2}\right) \cdot \psi_{0}\left(w_{1}, w_{2}\right)$; then the entire function $\psi\left(w_{1}, w_{2}\right)$ has the properties

$$
\begin{aligned}
& \psi\left(w_{1}+1, w_{2}\right)=\psi\left(w_{1}, w_{2}\right), \\
& \psi\left(w_{1}, w_{2}+1\right)=\operatorname{Exp}\left(-w_{1}\right) \cdot \psi\left(w_{1}, w_{2}\right) .
\end{aligned}
$$

${ }^{*}$ To simplify the typography, we shall use the notation $e^{2 \pi i x}=\operatorname{Exp}(x)$. 
Once more we introduce new variables by the equations

and write

$$
x=\operatorname{Exp}\left(w_{1}\right), \quad y=\operatorname{Exp}\left(w_{2}\right)
$$

$$
u(x, y)=\psi\left(w_{1}, w_{2}\right)=\psi\left(\frac{1}{2 \pi i} \log x, \frac{1}{2 \pi i} \log y\right) ;
$$

then $u(x, y)$ is holomorphic for all $x, y$ at finite distance, except $x=0$, $y=y$ and $x=x, y=0$. Starting with some definite branches of $\log x$ and $\log y$, say those that equal zero for $x=1$ and $y=1$ respectively, it follows from (4) that $u(x, y)$ is uniform in respect to $x$, while the analytic continuation along a path winding about $y=0$ once in the positive sense transforms the initial branch $u(x, y)$ into a new branch $\bar{u}(x, y)$ such that

$$
\bar{u}(x, y)=\frac{1}{x} u(x, y) .
$$

Now let us construct the function $G(x, y)$ of the lemma from the following data:

$S:$ the circular ring $\frac{1}{2}<|x|<2$;

$S^{\prime}$ : the circular ring $\frac{1}{2}<|y|<2$;

$R_{1}$ : the part of $S^{\prime}$ to the right of the imaginary axis;

$R_{2}$ : the part of $S^{\prime}$ to the left of the imaginary axis;

$l_{12}$ : the straight line segment from $y=2 i$ to $y=\frac{1}{2} i$;

$l_{12}^{\prime}$ : the straight line segment from $y=-\frac{1}{2} i$ to $y=-2 i$, so that the common

part of the boundaries of $R_{1}$ and $R_{2}$ consists of $l_{12}$ and $l_{12}^{\prime}$;

$u_{1}(x, y)$ : the initial branch of $u(x, y)$ defined above;

$u_{2}(x, y)$ : the analytic continuation of $u_{1}(x, y)$ across the line $l_{12}$.

Then $u_{1}(x, y)$ and $u_{2}(x, y)$ are uniform and holomorphic in $\left(S, R_{1}\right)$ and ( $S, R_{2}$ ) respectively, boundaries included. On $l_{12}$,

while on $l_{12}^{\prime}$ we have

$$
g_{12}(x, y)=\frac{u_{2}(x, y)}{u_{1}(x, y)}=1 \text {, }
$$

according to (7). We now make

$$
g_{12}^{\prime}(x, y)=\frac{u_{2}(x, y)}{u_{1}(x, y)}=\frac{1}{x}
$$

$$
G_{12}(x, y)=\log 1=0, \quad G_{12}^{\prime}(x, y)=-\log x,
$$

where that branch of the logarithm is taken which vanishes at $x=1$; since there are no vertices and therefore no integers $K_{b}$ to be determined, we may proceed at once to write down $\Phi(x, y)$ :

$$
\Phi(x, y)=\frac{1}{2 \pi i} \int_{-\frac{1}{2} i}^{-2 i} \frac{-\log x d z}{z-y}=\frac{1}{2 \pi i} \log x \cdot \log \frac{y+\frac{1}{2} i}{y+2 i},
$$


where the last logarithm is the branch that vanishes for $y$ infinite. Finally we obtain

$$
G(x, y)=u_{p}(x, y) \operatorname{Exp}\left(\frac{1}{2 \pi i} \cdot \frac{1}{2 \pi i} \log x \cdot \log \frac{y+\frac{1}{2} i}{y+2 i}\right)
$$

in $\left(S, R_{p}\right)$ for $p=1,2$. This $G(x, y)$ now has all the properties indicated in the lemma (as is also readily verified directly in this particular case). Nevertheless, $G(x, y)$ is not uniform in $\left(S, S^{\prime}\right)$, for letting $x$ describe a closed path in $S$ starting and ending at $x=1$, and winding about $x=0$ once in the positive sense, while $y$ describes a closed path interior to $R_{1}, \log x$ increases by $2 \pi i$, while $\log \left(y+\frac{1}{2} i\right) /(y+2 i)$ and $u_{1}(x, y)$ remain unchanged, and we arrive at a branch $\bar{G}(x, y)$ connected with the initial branch $G(x, y)$ by the relation

$$
\bar{G}(x, y)=\frac{y+\frac{1}{2} i}{y+2 i} G(x, y) .
$$

Hence Cousin's lemma, and with it his proofs of Theorem B, are valid when, and only when, not more than one of the regions $S_{1}, S_{2}, \cdots, S_{n}$ is multiply connected.

3. Example of a FunCtion of two VARIABles, MEROMORPHic IN A REgion $\left(S, S^{\prime}\right)$, WHICH CANNOT BE EXPRESSED AS THE QUOTIENT OF TWO RELATIVELY PRIME FUNCTIONS OF ENTIRE CHARACTER

From (3) it is evident that the quotient

$$
\frac{\psi_{1}\left(w_{1}, w_{2}\right)}{\psi_{0}\left(w_{1}, w_{2}\right)}=\frac{\phi_{1}\left(v_{1}, v_{2}\right)}{\phi_{0}\left(v_{1}, v_{2}\right)}
$$

is a meromorphic quadruply periodic function of $w_{1}$ and $w_{2}$ with the periods

$$
\begin{array}{cccc}
1, & 0, & -\frac{2 \tau_{22}}{\tau_{12}}, & \frac{2 \tau_{12}^{2}-2 \tau_{11} \tau_{22}}{\tau_{12}} \text { in } w_{1}, \\
0, & 1, \quad \frac{1}{\tau_{12}}, & \frac{\tau_{11}}{\tau_{12}} & \text { in } w_{2} .
\end{array}
$$

By (2), $\phi_{0}\left(v_{1}, v_{2}\right)$ contains only even, and $\phi_{1}\left(v_{1}, v_{2}\right)$ only odd, powers of Exp. $\left(v_{1}\right)$; hence these two functions are linearly independent, and the quotient considered is not a constant. Introducing the variables $x$ and $y$ by (5) and writing

$$
f(x, y)=\frac{\psi_{1}\left(w_{1}, w_{2}\right)}{\psi_{0}\left(w_{1}, w_{2}\right)}
$$

$f(x, y)$ is a non-constant, uniform function of $x$ and $y$, meromorphic in the region $\left(S, S^{\prime}\right)$, where $S$ consists of all points at finite distance in the $x$-plane, 
the point $x=0$ excepted, and $S^{\prime}$ is defined similarly in the $y$-plane. This function has the properties

$$
\begin{aligned}
& f(h x, k y)=f(x, y), \\
& f(l x, m y)=f(x, y),
\end{aligned}
$$

where

$$
\begin{gathered}
h=\operatorname{Exp}\left(-\frac{2 \tau_{22}}{\tau_{12}}\right), \quad k=\operatorname{Exp}\left(\frac{1}{\tau_{12}}\right), \\
l=\operatorname{Exp}\left(\frac{2 \tau_{12}^{2}-2 \tau_{11} \tau_{22}}{\tau_{12}}\right), \quad m=\operatorname{Exp}\left(\frac{\tau_{11}}{\tau_{12}}\right) .
\end{gathered}
$$

Now let us subject $\tau_{11}, \tau_{12}, \tau_{22}$ to the further condition that

$$
l^{a} m^{b} \neq h^{c} k^{d}
$$

for any integers $a, b, c$, and $d$ which are not all equal to zero. By (10), this is equivalent to the condition that the equation

$$
b \tau_{11}+n \tau_{12}+2 c \tau_{22}+2 a\left(\tau_{12}^{2}-\tau_{11} \tau_{22}\right)-d=0
$$

shall have no solution in integers $a, b, c, d, n$ which are not all equal to zero.* Then $f(x, y)$ cannot be expressed as the quotient of two relatively prime functions of entire character in $\left(S, S^{\prime}\right)$. For the purpose of an example, it is sufficient to carry out the proof in a special case, giving numerical values to $\tau_{11}, \tau_{12}, \tau_{22} \cdot \dagger$ Let us make

$$
\tau_{11}=i, \quad \tau_{12}=\frac{1}{\sqrt[ \pm]{2}}, \quad \tau_{22}=i \sqrt{2} ;
$$

then the real part of $2 \pi i\left(\tau_{11} n_{1}^{2}+2 \tau_{12} n_{1} n_{2}+\tau_{22} n_{2}^{2}\right)$ is $-2 \pi\left(n_{1}^{2}+\sqrt{2} n_{2}^{2}\right)$, a definite negative quadratic form in $n_{1}$ and $n_{2}$. Furthermore $\tau_{12} \neq 0$, and (12) gives upon separation of the real and imaginary parts

whence

$$
b+2 c \sqrt{2}=0, \quad n+3 a \sqrt[4]{8}-d \sqrt[4]{2}=0,
$$

$$
b=c=0, \quad n^{2}+12 a d-\left(18 a^{2}+d^{2}\right) \sqrt{2}=0, \quad a=d=n=0 .
$$

Hence (11) is satisfied, and in particular we have for any integers $\lambda$ and $\mu$, except $\lambda=\mu=0$,

$$
h^{\lambda} k^{\mu}-1 \neq 0, \quad l^{\lambda} m^{\mu}-1 \neq 0 .
$$

* In the theory of Theta functions, this condition expresses the fact that the period system $\tau_{11}, \tau_{12}, \tau_{22}$ is non-singular.

$\dagger$ This has the advantage of simplifying the convergence proof for the series (19). 
Now assume that $f(x, y)$ can be expressed in the form*

$$
f(x, y)=\frac{G_{1}(x, y)}{G_{0}(x, y)},
$$

where $G_{0}(x, y)$ and $G_{1}(x, y)$ are of entire character and relatively prime in $\left(S, S^{\prime}\right)$; we shall show that this leads to a contradiction. From (9) and (14) it follows that

$$
\frac{G_{0}(h x, k y)}{G_{0}(x, y)}=\frac{G_{1}(h x, k y)}{G_{1}(x, y)}, \quad \frac{G_{0}(l x, m y)}{G_{0}(x, y)}=\frac{G_{1}(l x, m y)}{G_{1}(x, y)},
$$

and since $G_{0}(x, y)$ and $G_{1}(x, y)$ are relatively prime, we conclude that both these quotients, which are evidently uniform, are holomorphic and different from zero in $\left(S, S^{\prime}\right) . \dagger$ Let us denote them by $g(x, y)$ and $g^{\prime}(x, y)$ respectively; then

$$
\begin{array}{r}
G_{\nu}(h x, k y)=g(x, y) G_{\nu}(x, y), \quad G_{\nu}(l x, m y)=g^{\prime}(x, y) G_{\nu}(x, y) \\
(\nu=0,1) .
\end{array}
$$

Since $g(x, y)$ is of entire character and different from zero in $\left(S, S^{\prime}\right)$, we may expand its logarithmic derivatives in Laurent's seriesł

$$
\frac{\partial \log g(x, y)}{\partial x}=\sum_{\lambda, \mu=-\infty}^{+\infty} a_{\lambda \mu} x^{\lambda} y^{\mu}, \quad \frac{\partial \log g(x, y)}{\partial y}=\sum_{\lambda,}^{+\infty} b_{\lambda \mu-\infty} x^{\lambda} y^{\mu},
$$

both series being absolutely and uniformly convergent for $\epsilon \leqq|x| \leqq 1 / \epsilon$, $\epsilon \leqq|y| \leqq 1 / \epsilon$, where $\epsilon$ is as small as we please. From

it follows that

$$
\frac{\partial^{2} \log g(x, y)}{\partial y \partial x}=\frac{\partial^{2} \log g(x, y)}{\partial x \partial y}
$$

$$
\sum \mu a_{\lambda \mu} x^{\lambda} y^{\mu-1}=\sum \lambda b_{\lambda \mu} x^{\lambda-1} y^{\mu},
$$

so that in particular $\mu a_{-1, \mu}=0, \lambda b_{\lambda_{1}-1}=0$, whence

$$
\begin{array}{lll}
a_{-1,0}=a, & a_{-1, \mu}=0 & (\mu \neq 0), \\
b_{0,-1}=b, & b_{\lambda,-1}=0 & (\lambda \neq 0) .
\end{array}
$$

* The following investigation is closely related to one made by Appell to an entirely different purpose in his paper Sur les fonctions périodiques de deux variables, J o u r nal d e M a thém a ti qu es, ser. 4, vol. 7 (1891), pp. 157-219. See pp. 185-201.

$\dagger$ This is a simple consequence of Weierstrass' preparation theorem; compare Cousin, l. c., $\S 15$, and Appell, l. c., pp. 182-185.

$\ddagger \mathrm{K}$. Weierstrass, Einige auf die Theorie der analytischen Funktionen mehrerer Veränderlichen sich beziehende Sätze, Mathematische Werke, vol. 2 (Berlin, 1895), pp. 135-188. See pp. 183188. 
Treating $g^{\prime}(x, y)$ in the same way, and integrating, we finally obtain

$$
\begin{aligned}
& g(x, y)=x^{a} y^{b} \operatorname{Exp}\left(\sum_{\lambda, \mu=-\infty}^{+\infty} A_{\lambda \mu} x^{\lambda} y^{\mu}\right), \\
& g^{\prime}(x, y)=x^{c} y^{d} \operatorname{Exp}\left(\sum_{\lambda, \mu=-\infty}^{+\infty} B_{\lambda \mu} x^{\lambda} y^{\mu}\right),
\end{aligned}
$$

the series being absolutely and uniformly convergent as before, and from the uniformity of $g(x, y)$ and $g^{\prime}(x, y)$ it is evident that $a, b, c, d$ are all integers. We arrive at a relation between $g(x, y)$ and $g^{\prime}(x, y)$ by observing that according to (15)

$$
\begin{gathered}
\frac{G_{\nu}(h l x, k m y)}{G_{\nu}(x, y)}=\frac{G_{\nu}(h l x, k m y)}{G_{\nu}(l x, m y)} \cdot \frac{G_{\nu}(l x, m y)}{G_{\nu}(x, y)}=g(l x, m y) g^{\prime}(x, y), \\
\frac{G_{\nu}(l h x, m k y)}{G_{\nu}(x, y)}=\frac{G_{\nu}(l h x, m k y)}{G_{\nu}(h x, k y)} \cdot \frac{G_{\nu}(h x, k y)}{G_{\nu}(x, y)}=g^{\prime}(h x, k y) g(x, y),
\end{gathered}
$$

whence

$$
g(l x, m y) g^{\prime}(x, y)=g^{\prime}(h x, k y) g(x, y) .
$$

Introducing the expressions (16) into this relation, we obtain $l^{a} m^{b} \operatorname{Exp}\left[\sum\left(A_{\lambda \mu} l^{\lambda} m^{\mu}+B_{\lambda \mu}\right) x^{\lambda} y^{\mu}\right]$

which evidently gives

$$
=h^{c} k^{d} \operatorname{Exp}\left[\sum\left(B_{\lambda \mu} h^{\lambda} k^{\mu}+A_{\lambda \mu}\right) x^{\lambda} y^{\mu}\right],
$$

$$
A_{\lambda \mu}\left(l^{\lambda} m^{\mu}-1\right)=B_{\lambda \mu}\left(h^{\lambda} k^{\mu}-1\right)
$$

and $l^{a} m^{b}=h^{c} k^{d}$. But in the last relation it follows from (11)-and this is the main point of the proof-that the integers $a, b, c$, and $d$ are all equal to zero. Moreover, (13) shows that we may write (17) in the form

$$
\frac{A_{\lambda \mu}}{h^{\lambda} k^{\mu}-1}=\frac{B_{\lambda \mu}}{l^{\lambda} m^{\mu}-1}, \quad \text { except for } \lambda=\mu=0 .
$$

Denote by $\sum^{\prime}$ a series from which the combination $\lambda=\mu=0$ is excluded, and write

$$
G(x, y)=\sum_{\lambda, \mu=-\infty}^{+\infty} \frac{A_{\lambda \mu}}{h^{\lambda} k^{\mu}-1} x^{\lambda} y^{\mu}=\sum_{\lambda, \mu=-\infty}^{+\infty} \frac{B_{\lambda \mu}}{l^{\lambda} m^{\mu}-1} x^{\lambda} y^{\mu}
$$

then (18) shows that the two definitions of $G(x, y)$ are formally consistent. For the convergence proof, separate the terms where $\lambda \neq 0$ from those with $\lambda=0$; we obtain with the aid of (18)

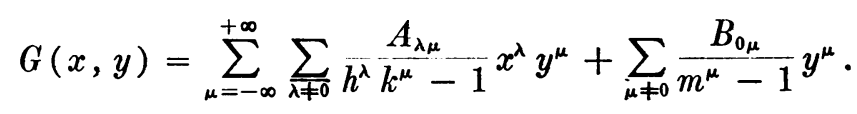


Introducing the numerical values of $\tau_{11}, \tau_{12}, \tau_{22}$ in (10), we find

$$
h=e^{4 \pi \sqrt[4]{2}}, \quad k=e^{2 \pi i \sqrt[4]{2}}, \quad m=e^{-2 \pi \sqrt[4]{2}},
$$

and consequently

$$
\left|h^{\lambda} k^{\mu}-1\right| \geqq\left.|| h\right|^{\lambda}|k|^{\mu}-1|=| e^{4 \pi \sqrt[4]{2} \cdot \lambda}-1 \mid ;
$$

the last expression being greater than $e-1$ or $1-e^{-1}$ according as $\lambda$ is a positive or negative integer, we have $\left|h^{\lambda} k^{\mu}-1\right|>\frac{1}{2}$ for $\lambda \neq 0$, and similarly $\left|m^{\mu}-1\right|>\frac{1}{2}$ for $\mu \neq 0$. Therefore (19) converges absolutely and uniformly in the same region as (16), that is, for $\epsilon \leqq|x| \leqq 1 / \epsilon, \epsilon \leqq|y| \leqq 1 / \epsilon$. Evidently $G(x, y)$ satisfies the relations

If we now write

$$
\begin{aligned}
& G(h x, k y)-G(x, y)=\sum^{\prime} A_{\lambda \mu} x^{\lambda} y^{\mu}, \\
& G(l x, m y)-G(x, y)=\sum^{\prime} B_{\lambda \mu} x^{\lambda} y^{\mu} .
\end{aligned}
$$

$$
G_{\nu}^{\prime}(x, y)=\operatorname{Exp}[-G(x, y)] \cdot G_{\nu}(x, y) \quad(\nu=0,1),
$$

$G_{0}^{\prime}(x, y)$ and $G_{1}^{\prime}(x, y)$ are of entire character (and relatively prime) in $\left(S, S^{\prime}\right)$, and by (14)

$$
f(x, y)=\frac{G_{1}^{\prime}(x, y)}{G_{0}^{\prime}(x, y)}
$$

From (15), (16), and (20) we find, bearing in mind that $a=b=c=d=0$,

$$
\begin{aligned}
& G_{\nu}^{\prime}(h x, k y)=\operatorname{Exp}\left(A_{\mathrm{co}}\right) \cdot G_{\nu}^{\prime}(x, y), \\
& G_{\nu}^{\prime}(l x, m y)=\operatorname{Exp}\left(B_{00}\right) \cdot G_{\nu}^{\prime}(x, y)
\end{aligned}
$$

Expanding $G_{0}^{\prime}(x, y)$ and $G_{1}^{\prime}(x, y)$ in Laurent's series

$$
G_{0}^{\prime}(x, y)=\sum_{\lambda, \mu=-\infty}^{+\infty} C_{\lambda \mu} x^{\lambda} y^{\mu}, \quad G_{1}^{\prime}(x, y)=\sum_{\lambda, \mu=-\infty}^{+\infty} D_{\lambda \mu} x^{\lambda} y^{\mu},
$$

the first equation (22) gives

$$
C_{\lambda \mu}\left[h^{\lambda} k^{\mu}-\operatorname{Exp}\left(A_{00}\right)\right]=D_{\lambda \mu}\left[h^{\lambda} k^{\mu}-\operatorname{Exp}\left(A_{00}\right)\right]=0 .
$$

Since $G_{0}^{\prime}(x, y)$ is not identically zero, one $C_{\lambda \mu}$ at least must be different from zero, say $C_{\rho \sigma}$, so that $h^{\rho} k^{\sigma}-\operatorname{Exp}\left(A_{00}\right)=0$. If $h^{\lambda} k^{\mu}-\operatorname{Exp}\left(A_{00}\right)=0$, it follows that $h^{\lambda-\rho} k^{\mu-\sigma}-1=0$, whence $\lambda=\rho, \mu=\sigma$ by (13). Therefore $h^{\lambda} k^{\mu}-\operatorname{Exp}\left(A_{00}\right) \neq 0$, and $C_{\lambda \mu}=D_{\lambda \mu}=0$ except for $\lambda=\rho, \mu=\sigma$, and (21) gives

$$
f(x, y)=\frac{D_{\rho \sigma} x^{\rho} y^{\sigma}}{C_{\rho \sigma} x^{\rho} y^{\sigma}}=\text { const. }
$$

But we have seen from the definition of $f(x, y)$ that this function is not a constant, and this contradiction shows that Theorem $\mathbf{C}$ (and consequently 
Theorem B, since B implies C) is not valid when two of the regions $S_{1}, S_{2}$, $\cdots, S_{n}$ are multiply connected.

It is possible however to express our function $f(x, y)$ as the quotient of two functions $G_{1}(x, y)$ and $G_{0}(x, y)$ of entire character in $\left(S, S^{\prime}\right)$, if we remove the condition that these two functions shall be relatively prime. To prove this, let $\rho=0$ or 1 and write

$$
\psi_{2}\left(w_{1}, w_{2}\right)=\operatorname{Fxp}\left(2 \tau_{22} w_{2}^{2}\right) \cdot \psi_{\rho}\left(w_{1},-w_{2}\right) ;
$$

it then follows from (3) that

$$
\begin{aligned}
& \psi_{2}\left(u_{1}+1, w_{2}\right)=\psi_{2}\left(w_{1}, w_{2}\right), \\
& \psi_{2}\left(w_{1}, w_{2}+1\right)=\operatorname{Exp}\left(w_{1}+2 \tau_{22} w_{2}+\tau_{22}\right) \psi_{2}\left(w_{1}, w_{2}\right),
\end{aligned}
$$

so that

$$
\begin{aligned}
& \psi_{2}\left(w_{1}+1, w_{2}\right) \psi_{\nu}\left(w_{1}+1, w_{2}\right)=\psi_{2}\left(w_{1}, w_{2}\right) \psi_{\nu}\left(w_{1}, w_{2}\right), \\
& \psi_{2}\left(w_{1}, w_{2}+1\right) \psi_{\nu}\left(w_{1}, w_{2}+1\right)=\psi_{2}\left(w_{1}, w_{2}\right) \psi_{\nu}\left(w_{1}, w_{2}\right)
\end{aligned}
$$

and consequently, writing

$$
G_{\nu}(x, y)=\psi_{2}\left(w_{1}, w_{2}\right) \psi_{\nu}\left(w_{1}, w_{2}\right) \quad(\nu=0,1),
$$

$G_{0}(x, y)$ and $G_{1}(x, y)$ are both uniform functions of $x$ and $y$, holomorphic in $\left(S, S^{\prime}\right)$. Since $f(x, y)=\psi_{1}\left(w_{1}, w_{2}\right) / \psi_{0}\left(w_{1}, w_{2}\right)$, we have in

$$
f(x, y)=\frac{G_{1}(x, y)}{G_{0}(x, y)}
$$

a representation of $f(x, y)$ of the required character. Evidently $G_{0}(x, y)$ and $G_{1}(x, y)$ have here the common manifold of zeros defined by

$$
\psi_{2}\left(w_{1}, w_{2}\right)=0,
$$

and from what we have proved before regarding $f(x, y)$, it follows that the common divisor cannot be removed without destroying the uniformity of $G_{0}(x, y)$ and $G_{1}(x, y)$.

In a subsequent paper, it will be shown that this representation as the quotient of two functions of entire character with common divisor is possible for any function $f(x, y)$, meromorphic everywhere at finite distance except at the points defined by $G(x, y)=0$, where $G(x, y)$ is an entire function. The common divisor cannot in general be removed except when $G(x, y)$ is irreducible. 